\title{
Bioinformed Design of Dynamic Tensegrity Units
}

\author{
Cristhian Castro-Arenas \\ Centro Laboratorio de Morfología, Instituto de la Espacialidad Humana (IEH), Facultad de \\ Arquitectura, Diseño y Urbanismo, Universidad de Buenos Aires; Laboratorio de \\ Biomecánica e Ingeniería para la Salud (LaBIS), Facultad de Ingeniería y Ciencias Agrarias, \\ Pontificia Universidad Católica Argentina. | Argentina | ccastroarenas@uca.edu.ar

\section{Mónica Miralles} \\ Centro de Investigación en Diseño Industrial de Productos Complejos (CIDI), Facultad de \\ Arquitectura, Diseño y Urbanismo, Universidad de Buenos Aires; Laboratorio de \\ Biomecánica e Ingeniería para la Salud (LaBIS), Facultad de Ingeniería y Ciencias Agrarias, \\ Pontificia Universidad Católica Argentina. | Argentina | mmiralles@uca.edu.ar
}

\begin{abstract}
This paper presents the bioinformed design of tensegrities based in the application of configurative logics of biotensegrities. Its purpose is to accomplish dynamic tensegrities, potentially applicable in the design of innovative technological devices. This article presents the analysis and design of three types of models: a) the Universal Tensegrity Joints introduced by Fuller, b) the Abstract Dynamic Units, and c) Bioinformed Dynamic Units. The methodology is based on simulating movements with parametric modeling in Rhinoceros software, with the usage of Grasshopper and Kangaroo plugins. Thus, a first classification of UDAs and the first phase of UDB models for leg and shoulder were obtained.
\end{abstract}

Keywords: Tensegrity; Biotensegrity; Bioinformed; Parametric; Design.

\section{INTRODUCCIÓN}

El diseño biológicamente informado se caracteriza por utilizar hallazgos de la investigación biológica para informar la búsqueda de soluciones de diseño. Su objetivo es la innovación y puede, o no, dar lugar a soluciones sostenibles (louguina, Dawson, Hallgrimsson, \& Smart, 2014). En este sentido, llamamos tensegridad bioinformada a aquella en cuyo proceso de diseño y concreción, se incluye información biológica, abarcando, incluso, los métodos de búsqueda de forma, los materiales y/o los métodos de fabricación.

La tensegridad, principio estructural natural basado en el equilibrio de fuerzas opuestas, ha sido aplicada en el diseño mediante la tecnotraducción de formas cristalinas debido a su alto grado de simetría (Emmerich, 1992; Fuller, 1961). Si bien, estas células son ampliamente conocidas y estudiadas, la búsqueda de otras formas estables de tensegridad es un problema abierto. A pesar del dinamismo inherente en este sistema, su potencial transformativo ha sido poco explorado.

Este trabajo aborda el diseño bioinformado de articulaciones de gran movilidad (diartrosis) a partir de una estrategia secuencial que parte de dos modelos de tensegridad de Fuller y de su parametrización correspondiente. A esta etapa sigue la de las Unidades Dinámicas Abstractas, que corresponde a la generación de células dinámicas genéricas con un fuerte potencial de adaptación. Finalmente, la tercera etapa, corresponde a las Unidades Dinámicas Bioinformadas. Unidades surgidas de la modificación de las anteriores, que incluyen información biológica de carácter anátomo-funcional.

Todo ello dio origen a una primera generación de Unidades Dinámicas Bioinformadas, enfocadas en las articulaciones de la rodilla y el hombro, utilizando herramientas digitales propias del diseño.

Para fundamentar las analogías y, como punto de partida, se revisa, a continuación, la tensegridad como principio natural y la tensegridad en relación al movimiento.

\section{TENSEGRIDAD COMO PRINCIPIO NATURAL}

Al igual que las producciones naturales, la tensegridad remite al principio de relación estructural que propicia la configuración de sistemas morfoestructurales multiescala cuyos componentes, dedicados a transmitir fuerzas opuestas, corresponden a elementos comprimidos aislados, integrados por una red de elementos tensionados (Fuller \& Applewhite, 1975). En analogía con la distribución espacial de las fuerzas de atracción y repulsión entre átomos o moléculas, las fuerzas de tensión-compresión condicionan directamente la distribución de masa del sistema y la dinámica de su comportamiento en una dada configuración (Ingber, 2018; Hensel \& Menges, 2008).

Desde un plano proyectual tecnomorfológico, un sistema básico de tensegridad estará conformado por la mínima cantidad de componentes que aseguren la autoestabilidad mediante este principio (Reissig, 2012). Estos sistemas mínimos son conocidos como simplex o equilibriums. Los 
más estudiados son aquellos cuya morfología es similar a la de configuraciones cristalinas (Emmerich, op. cit.; Motro, 2003). Ello se debe a que, su alto grado de simetría, garantiza el equilibrio estable y facilita su fabricación. Es por ello que, gran parte de las tensegridades conocidas son estáticas, pudiendo, en algunos casos, tener respuestas adaptativas y reaccionar a estímulos externos.

Entre estos sistemas mínimos encontramos los antiprismas y los sólidos platónicos (Figura 1), que tienen la cualidad de funcionar individualmente, o agruparse, para configurar sistemas más complejos. Esta cualidad modular remite al comportamiento celular $y$, desde un enfoque bioinformado, a su capacidad de conformar tejidos especializados. En este trabajo denominaremos células a los sistemas mínimos y tejidos, a sus agrupaciones.

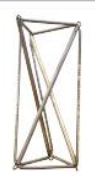

a

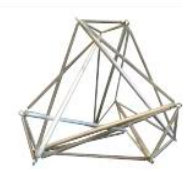

b

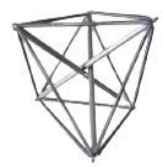

C
Figura 1: Diferentes células de tensegridad:

a) trípode o antiprisma de base $3, b$ ) tetraedro truncado, c) octaedro por vector interno.

\section{TENSEGRIDAD Y MOVIMIENTO}

La tensegridad presenta un comportamiento dinámico constante debido a la red tensada que la integra. Esta característica ha sido objeto de estudio por su potencial aplicación en el diseño de metamateriales y dispositivos tecnológicos de diferente índole. El análisis y diseño de sus movimientos ha sido abordado desde el comportamiento de células individuales, tejidos homogéneos y heterogéneos y configuraciones de diferente complejidad, como las mostradas en las investigaciones de Rimoli, \& Pal (2017), Castro-Arenas, Ghersi, Borsoi, \& Miralles (2017); SunSpiral V., Agogino A., \& Atkinson D. (2015); Friesen, Pogue, Bewley, de Oliveira, Skelton, \& Sunspiral (2014) y Aldrich, Skelton, \& Kreutz-Delgado (2003), entre otros.

Estas investigaciones han permitido entender algunos problemas asociados al equilibrio dinámico, a la dificultad para controlar sus transformaciones y a la complejidad motriz debida a la coordinación conjunta de grupos de tensores, o bien, de compresores sujetos a complejos sistemas de control (Pajunen, 2020; González \& Luo, 2019; Khazanov, Humphreys, Keat, \& Rieffel, 2013; Skelton, 2005).

En su libro Synergetics, Fuller (op. cit.) analiza diferentes modelos de tensegridad en su versión mínima, tanto naturales como artificiales. En estos últimos, a los que llama Articulaciones Universales, identifica dos modelos que propician el movimiento en diferentes ejes, los cuales son la base de nuestro estudio (ver Figura 2).

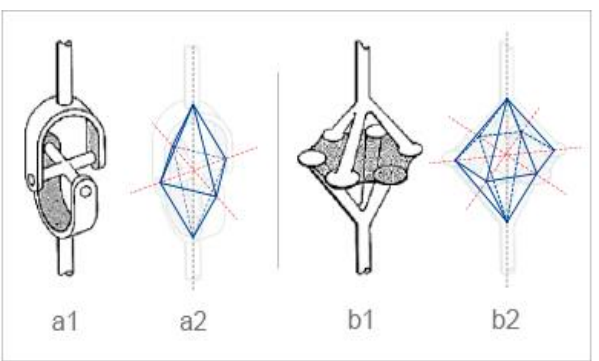

Figura 2: Articulaciones Universales (AU) propuestas por Fuller. a1 y b1) AU de dos y tres ejes (versiones originales), a2 y b2) abstracción geométrica.

(Adaptado de Fuller \& Applewhite, 1975.)

\section{TENSEGRIDAD Y BIOMECANICA: BIOTENSEGRIDAD}

El nacimiento de la biotensegridad estimuló un cambio de enfoque en el diseño de tensegridades. Desde este nuevo paradigma biomecánico se analizan aspectos morfológicos y performativos de diversos sistemas locomotrices orgánicos que exhiben características propias de la tensegridad (Scarr, 2014; Swanson, 2013; Ingber, 1993, 2003; Levin, 1982, 1991, 2006).

Estas tensegridades biológicas se convirtieron entonces, en nuevos modelos para ser analizados con objetivos proyectuales ligados a la innovación tecnológica (Cretu, 2011, 2009). Esta nueva estrategia de diseño estimuló investigaciones y proyectos como los desarrollados por Jung, E., Ly, V., Cessna, N., Ngo, M. L., Castro, D., SunSpiral, V., \& Teodorescu, M. (2018) y Lessard, S., Bruce, J., Jung, E., Teodorescu, M., SunSpiral, V., \& Agogino, A. (2016), entre otros.

En estas investigaciones se presentan robots que emulan, de manera abstracta, las formas y movimientos de extremidades humanas. Allí, los modelos son conformados con elementos lineales que exhiben características similares a las articulaciones universales de la Figura 2, aunque no presentan una profunda correspondencia anatómica. En el presente trabajo, para lograr una emulación biomecánica más cercana para cada tensegridad, el abordaje bioinformado considera los accidentes óseos y las inserciones musculares como puntos nodales que definen, tanto las formas de los compresores, como los nudos de conexión con los tensores.

\section{METODOLOGÍA}

Ésta estrategia para el diseño de tensegridades bioinformadas se centra en el análisis y diseño de tres tipos de modelos a través de una secuencia que contempla: a) el análisis cuali-cuantitativo de las Articulaciones Universales (AU) de tensegridad de 2 y 3 ejes, b) el diseño de Unidades Dinámicas Abstractas (UDAs), y c) el diseño de Unidades Dinámicas Bioinformadas (UDBs). Cada una de estas etapas contó con el modelado paramétrico en el software Rhinoceros, permitiendo la simulación de sus movimientos con los plugins Grasshopper y Kangaroo. 


\section{A) ARTICULACIÓN UNIVERSAL DE 2 Y 3 EJES}

Los dos sistemas de tensegridad presentados por Fuller como AU pueden ser definidos como células independientes cuya configuración propicia, tanto su integridad tensional, como el dinamismo potencialmente dirigido.

Desde un punto de vista morfológico estas dos articulaciones pueden ser entendidas como pirámides que se conectan por su base. En el primer caso son dos pirámides de base cuadrada -generando un octaedro- y en el segundo, de base hexagonal. Los ejes que las definen son aquellos que coinciden con las aristas de su base, como se observa en la Figura 2 (líneas punteadas rojas).

Los componentes que soportan la compresión corresponden a aristas de las dos pirámides que conforman cada célula. Su materialización alternada (no continua) es la que la convierte en un sistema articulado que se puede clasificar como una tensegridad Clase I (Skelton, Helton, \& Adhikari, 1998). Esta característica garantiza su estabilidad y les otorga amplio rango de movimientos en diferentes ejes.

La AU de 2 ejes corresponde al sistema mecánico conocido como junta cardán, usado históricamente en el árbol de transmisión de algunos vehículos y otras máquinas. Al hacer su traducción a un sistema clásico de tensegridad (lineal) se hace necesario reemplazar la pieza central, que conecta las partes superior e inferior (cruceta), por tensores que conectan los extremos de los compresores (horquillas). También se hace necesario sumar tensores para lograr la estabilización del sistema de tal manera que funcione como una célula independiente, como se observa en la Figura 3a.

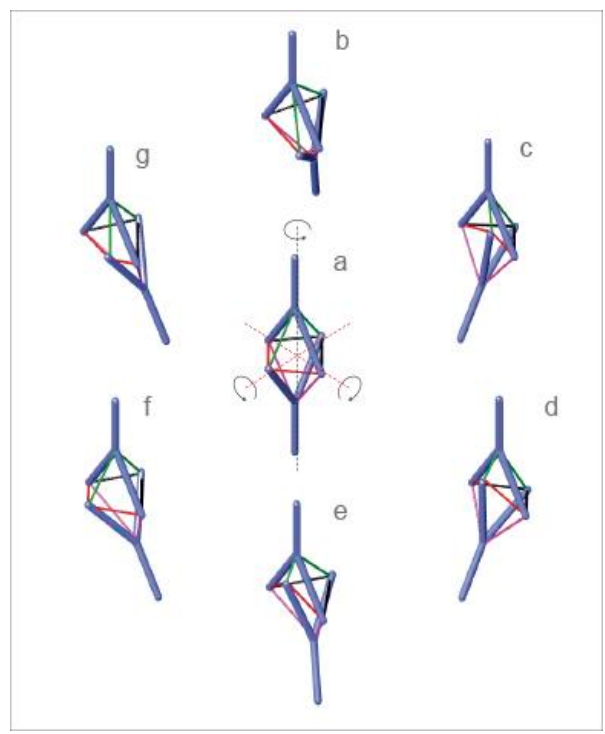

Figura 3: Transformaciones de la UDA 2-2 mediante el acortamiento de los tensores activos. En cada imagen los tensores activos han sido reducidos al $20 \%$ de su longitud total según la rotación deseada.

Se observa: a) En reposo, b) rotación en z, c) rotación $z$ - $y$, d) rotación en $y, e$ ) rotación en $x-y$, f) rotación en $x$, g) rotación en $x-z$.
La AU de 3 ejes es una propuesta teórica de Fuller. En este caso su traducción se ha hecho de manera similar a la anterior, reemplazando la membrana central por tensores lineales y sumando cables estabilizadores. En ambos casos los tensores pueden ser reemplazados por membranas, tal como sucede con cualquier sistema de tensegridad, pero han sido simplificados a tensores rectilíneos para esta fase de estudio.

\section{PARAMETRIZACIÓN DIGITAL}

La estructura fue dividida en tres partes para su análisis y parametrización. Las dos primeras corresponden a los compresores (superior e inferior) y la tercera a los tensores. Cada parte fue definida mediante el uso de componentes de las categorías Params, Maths, Vector y Curve permitiendo, de manera sencilla, la parametrización de los puntos y planos relativos de las formas básicas que definen su configuración. Esto se logra mediante el uso de círculos y planos relativos, asociados a tamaños, ángulos, divisiones y a lugares de unión. A su vez, estos puntos definen los extremos de las formas lineales de los compresores y los puntos de conexión con los tensores.

Cada parte cuenta con su propia definición (conjunto de baterías) lo cual permite su alteración individual. Al modificar estos valores, las operaciones se ajustarán automáticamente al nuevo modelo, permitiendo la visualización de las variaciones morfológicas y su comportamiento en tiempo real. Los tensores han sido parametrizados de manera individual permitiendo su modificación independiente como tensores pasivos y/o activos (actúan como ligamentos y músculos, respectivamente).

El comportamiento dinámico de estas tensegridades ha sido testeado como un sistema colgante, anclado en el punto extremo del compresor superior, permitiendo activar sus tensores y observar sus transformaciones de manera libre. Las fuerzas aplicadas con el plugin Kangaroo asignan resistencia a la deformación en los componentes, además de aplicar gravedad al sistema. En la Figura 3 podemos observar la simulación de la UDA 2-2.

\section{B) UNIDADES DINÁMICAS ABSTRACTAS (UDAs)}

Partiendo de las articulaciones universales como modelos fundamentales se desarrolló una variedad de opciones de manera sistemática, conservando su lógica formal y obteniendo células con diferentes características morfológicas y dinámicas. Las configuraciones diseñadas de esta manera han sido llamadas Unidades Dinámicas Abstractas (UDAs) debido a que su primordial objetivo es dinámico y su forma aún no está definida por alguna función o información biológica específica. Son combinaciones de pirámides con bases de polígonos equiláteros, intersectados por dicha cara poligonal (Figura 4). En síntesis, las UDAs son células dinámicas genéricas con el potencial de ser modificadas de acuerdo a objetivos específicos de diseño, como ya fue anticipado.

Los parámetros fijos que definen las UDAs son: el diámetro que inscribe el polígono base, la altura del ápice de la pirámide y el radio de los elementos compresores y tensores. Las simulaciones dinámicas del sistema dependen de la fuerza y elasticidad del conjunto de tensores en respuesta a la elongación y contracción de los 
tensores activos. En vista de que no tiene asociados materiales ni cargas específicas de diseño, este parámetro se ha establecido como unidad (valor 1) de acuerdo al comportamiento observado. Los movimientos de toda la estructura son producidos por la variación de la longitud del tensor deseado. En la Figura 3 se observan 6 transformaciones generadas por la reducción al $20 \%$ de 1 tensor (b, d, f) y de 2 tensores (c, e, g). El potencial de movimientos posibles en las UDAs dependerá del patrón generado por la cantidad y posición de tensores activados y sus porcentajes de contracción y/o elongación.

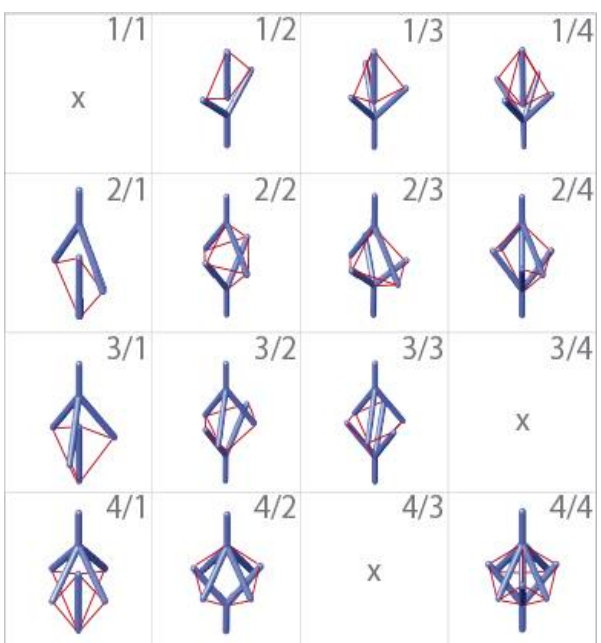

Figura 4: Clasificación 1.0 de Unidades Dinámicas Abstractas Se presenta una selección de la tabla combinatoria de las posibilidades de generación de UDAs de acuerdo a la cantidad de extensiones de cada componente compresor. La nomenclatura asociada da cuenta de la posición (arriba/abajo) y cantidad de extensiones del compresor indicado. Las X indican que con estos parámetros existe cruce de compresores, por lo tanto, no se tomaron en cuenta en esta selección.

\section{MODELOS FÍSICOS}

Con el fin de comprobar físicamente el comportamiento de las UDAs se fabricaron modelos con las características morfológicas anteriormente descriptas. Se presenta en este trabajo una primera versión de los resultados. Para los componentes a compresión se usaron barras cilíndricas de madera de pino de $\$ 5 \mathrm{~mm}$ y $150 \mathrm{~mm}$ de longitud, que fueron unidas mediante nudos impresos en PLA, diseñados según cada caso. Para los componentes a tensión se usaron bandas elásticas de látex de $Q 40 \mathrm{~mm}$ y $\pm 2 \mathrm{~mm}$ de sección cuadrada. También se imprimieron elementos flexibles en TPU para los tensores intermedios (Figura 5).

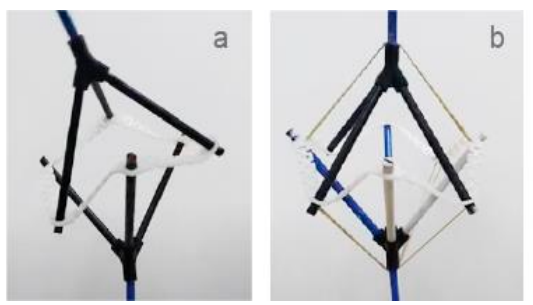

Figura 5: capturas de vídeo de las pruebas dinámicas de prototipos de UDAs: a) UDA 3-3 (sólo con tensores intermedios),

b) UDA 4-4 (con tensores intermedios y perimetrales)

\section{C) ,UNIDADES DINÁMICAS BIOINFORMADAS (UDBs)}

La inclusión de bioinformación en las UDAs, da nacimiento a las Unidades Dinámicas Bioinformadas (UDBs). Éstas surgen de la modificación configurativa de las UDAs, según la información de morfologías biológicas específicas pudiendo, a su vez, estar compuestas por combinaciones de varias UDAs. Los casos aquí presentados son una primera generación de modelos de tensegridad que corresponden a tecnotraducciones de articulaciones y segmentos de la pierna y el brazo (biotensegridades modelo).

\section{El proceso de bioinformación consistió en:}

(a) El estudio anatómico de las articulaciones de dichas extremidades, teniendo en cuenta músculos, tendones y ligamentos que intervienen en el movimiento, junto a la posición de los accidentes óseos correspondientes a las inserciones tendinosas.

(b) Selección y adaptación de las UDAs para lograr una tecnotraducción coherente con la articulación bajo estudio. Cada elemento anatómico tiene su homólogo en el sistema de tensegridad. Los compresores traducen a los huesos, los tensores activos a los músculos y los tensores pasivos modelan a los ligamentos y tendones.

\section{CASO 1: UDB PIERNA}

El primer paso fue identificar y definir los diferentes componentes del sistema que luego serían traducidos como compresores (huesos), tensores activos (músculos) y tensores pasivos (ligamentos y tendones). De esa manera se identificó al conjunto óseo que participa en el movimiento de flexión y extensión de la rodilla. El mismo está comprendido por la cadera, el fémur, la rótula, la tibia y el peroné. En cuanto a los tejidos blandos, que intervienen como tensores activos en el movimiento de flexión, se incluyó a los músculos: sartorio, bíceps femoral, grácil, gastrocnemio, semimembranoso y semitendinoso. Por su parte, los tejidos blandos, que intervienen pasivamente en estos movimientos, fueron: el ligamento iliofemoral, ligamento pubofemoral (en la cadera), el ligamento anterolateral, colateral-lateral y el ligamento rotuliano (rodilla) y la membrana interósea entre la tibia y el peroné.

Una vez identificados, se procedió a abstraer sus formas, de tal manera que su complejidad orgánica fuese asimilable con las formas rectilíneas de las UDAs. Para esto se tomaron en cuenta los puntos de inserción como extremos de las diferentes líneas rectas. Si bien, la forma de cada componente anatómico influye en el comportamiento estructural y dinámico, fueron abstraídos a su mínima expresión para esta fase del estudio.

La tecnotraducción de los huesos en elementos a compresión tuvo diferentes etapas. Primero se identificaron los puntos de inserción de los tejidos blandos en los huesos para conectar de manera similar los componentes correspondientes. Algunos de los puntos de inserción fueron simplificados promediando su posición, tales como la inserción del gastrocnemio y del ligamento colateral medial en el fémur (punto f1), los puntos de inserción del gastrocnemio y del ligamento colateral-lateral (punto f2), el sartorio y el recto femoral (punto c1). Los 
demás puntos corresponden a los cambios de dirección notables de los huesos y a puntos de inserción aislados y definidos. La traducción de los huesos con sus puntos de inserción, han sido identificados en las Tablas 1 y 2. Su abstracción gráfica como UDB se presenta en la Figura 6.

El extremo superior del compresor $\mathrm{C} 1$ adquiere cierta complejidad producida al abstraer las formas del hueso de la cadera. Ello es necesario para conectar los tensores activos y emular los movimientos bajo estudio. En el compresor inferior (C2), la tibia y el peroné fueron fusionados en un solo elemento, incluyendo también a la rótula en su extremo frontal superior. Estos análisis permitieron establecer como modelo base a la UDA 2-3, aplicando en ella las adaptaciones morfológicas pertinentes para obtener la UDB_pierna.

Los ligamentos fueron identificados y abstraídos como tensores pasivos para emular, en forma realista, su función cinemática, usando como puntos de inserción los anteriormente obtenidos. Dichos componentes corresponden al ligamento anterolateral, I. colateral medial y lateral, I. rotuliano y al I. cruzado anterior.

Los músculos, involucrados en los movimientos de flexión y extensión de la pierna, fueron traducidos como tensores activos (fusionando y/o simplificando con líneas rectas sus formas y recorridos). Para la flexión, el gastrocnemio y el tendón de Aquiles fueron vinculados en un mismo punto de inserción inferior, conectando así el talón con el fémur. El sartorio y el grácil, que conectan la cadera y la tibia, conectan en nuestro modelo puntos similares, modificando su forma "espiral" a una línea recta, cada uno con sus puntos de inserción correspondientes. Los músculos semimembranoso y semitendinoso fueron fusionados en un solo tensor activo, teniendo en cuenta su posición y función cooperativa en la flexión de la pierna. El bíceps femoral también fue abstraído en una línea recta, conectando los puntos análogos a las inserciones en el peroné y la cadera. Todos los componentes blandos del sistema, traducidos como tensores pasivos y activos, han sido clasificados y nomenclados en la Tabla 2.

Cada elemento fue modelado y definido digitalmente según su posición y rol estructural. Se asignó a cada línea una función de geometría independiente. Esto permitió la modificación, de manera individual o grupal, tanto de las fuerzas como de las longitudes, de acuerdo al movimiento deseado.

Tabla 1: Nomenclatura de compresores en la UDB_pierna. Las columnas corresponden al nombre anatómico, la correspondiente nomenclatura asignada en la UDB y los puntos de inserción identificados en cada compresor.

\begin{tabular}{|c|c|c|}
\hline Nom. anatómico & Nomen. UDB & P. de inser. ident. \\
\hline Cadera, fémur & C1 & $\begin{array}{c}c 1, c 2, c 3, \\
f 1, f 2, f 3, f 4\end{array}$ \\
\hline Rótula, tibia, peroné & $\mathrm{C} 2$ & $\mathrm{t} 1, \mathrm{t} 2, \mathrm{t} 3, \mathrm{t} 4, \mathrm{t} 5$ \\
\hline
\end{tabular}

Tabla 2: Nomenclatura de tensores pasivos y activos en la UDB_pierna. Las columnas corresponden al nombre anatómico, la correspondiente nomenclatura asignada en la UDB y los puntos de inserción a los cuales se conectan.

\begin{tabular}{|c|c|c|c|}
\hline $\begin{array}{c}\text { Tipo } \\
\text { tensores }\end{array}$ & Nom. anatómico & $\begin{array}{l}\text { Nomen. } \\
\text { UDB }\end{array}$ & $\begin{array}{l}\text { P. de inserc. } \\
\text { conec. }\end{array}$ \\
\hline \multirow{4}{*}{$\begin{array}{l}\text { Tensores } \\
\text { pasivos }\end{array}$} & $\begin{array}{l}\text { Ligamento } \\
\text { colateral - medial }\end{array}$ & L1 & $f 1-t 2$ \\
\hline & $\begin{array}{l}\text { L. anterolateral y } \\
\text { L. colateral-lateral }\end{array}$ & L2 & $f 2-t 4$ \\
\hline & $\begin{array}{l}\text { Ligamento } \\
\text { cruzado anterior }\end{array}$ & L3 & $f 3-t 1$ \\
\hline & $\begin{array}{l}\text { Ligamento } \\
\text { rotuliano }\end{array}$ & L4 & $f 2-t 5$ \\
\hline \multirow{8}{*}{$\begin{array}{c}\text { Tensores } \\
\text { activos }\end{array}$} & M. Recto femoral & E1 & $\mathrm{c} 1-\mathrm{t} 5$ \\
\hline & $\begin{array}{l}\text { M. Vasto lateral y } \\
\text { vasto medial }\end{array}$ & E2 & $f 4-t 5$ \\
\hline & M. gastrocnemio & $\mathrm{A} 1$ & $f 2-t 3$ \\
\hline & Talón de Aquiles & $\mathrm{A} 2$ & $f 1-t 3$ \\
\hline & M. Sartorio & $\mathrm{A} 3$ & $\mathrm{t} 2-\mathrm{c} 1$ \\
\hline & M. Grácil & A4 & $\mathrm{t} 2-\mathrm{c} 2$ \\
\hline & $\begin{array}{c}\text { M. Semitendinoso } \\
y \\
\text { semimembranoso }\end{array}$ & A5 & $\mathrm{t} 2-\mathrm{c} 3$ \\
\hline & $\begin{array}{l}\text { M. Bíceps femoral } \\
\text { de cabeza larga }\end{array}$ & $\mathrm{A} 6$ & $\mathrm{t} 4-\mathrm{c} 3$ \\
\hline
\end{tabular}

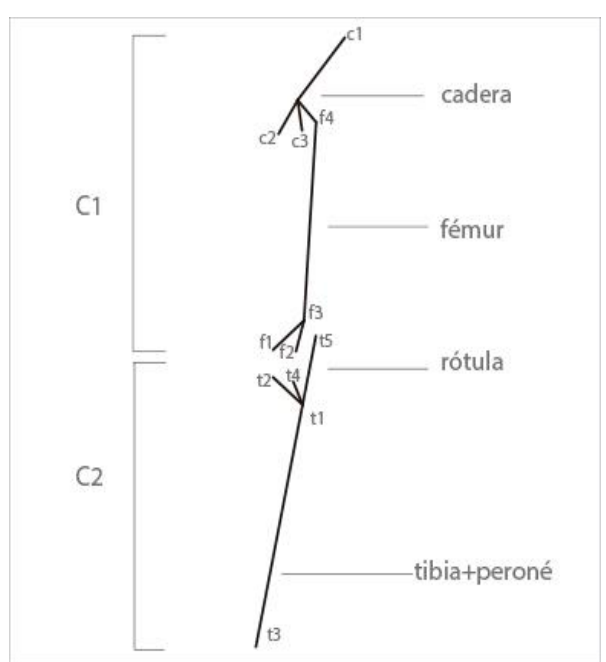

Figura 6: Abstracción de los huesos de la pierna en su traducción a componentes de la UDB pierna. Se observan los componentes compresores (en negro) y los puntos de inserción correspondientes.

\section{CASO 2: UDB_HOMBRO}

El proceso de abstracción en este caso fue similar al descripto para el Caso I, traduciendo las características morfofuncionales orgánicas específicas a la tecnomorfología correspondiente con las simulaciones y materializaciones de estas tensegridades. 
En los movimientos del hombro la cantidad de huesos en los que se insertan los músculos y tendones es mayor a los involucrados en el Caso 1. Estos huesos incluyen al sacro, las vértebras L5 a T8, siete pares de costillas y el esternón, además de la clavícula, la escápula y el húmero (Tabla 3 y Figura 7).

Tabla 3: Nomenclatura de compresores en la UDB_hombro. Las columnas corresponden al nombre anatómico, la correspondiente nomenclatura asignada en la UDB y los puntos de inserción identificados en cada compresor.

\begin{tabular}{ccc}
\hline Nom. anatómico & $\begin{array}{c}\text { Nom. } \\
\text { UDB }\end{array}$ & P. inserc. ident. \\
\hline $\begin{array}{c}\text { Clavícula, esternón, } \\
\text { escápula, col. dorsal }\end{array}$ & $\mathrm{C} 1$ & $\begin{array}{c}\mathrm{ac1}, \mathrm{c} 1, \mathrm{~d} 1, \mathrm{e} 1, \\
\mathrm{ec} 1-\mathrm{ec} 5,\end{array}$ \\
Húmero & $\mathrm{C} 2$ & $\mathrm{~h} 1-\mathrm{h} 10$ \\
\hline
\end{tabular}

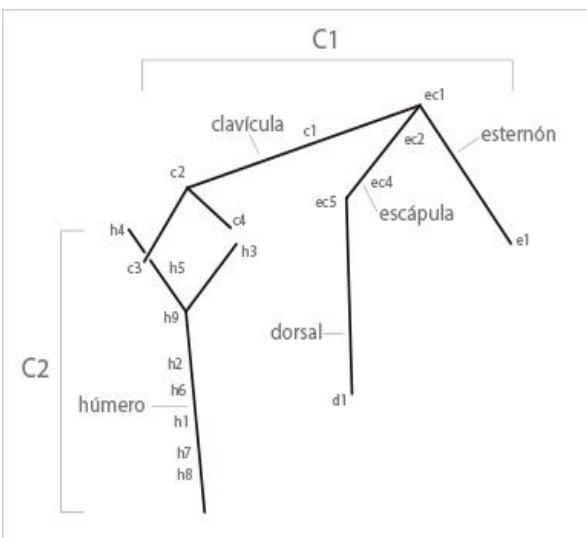

Figura 7: Abstracción de los huesos en su traducción a componentes de la UDB_hombro. Se observan los componentes compresores (en negro) y los puntos de inserción correspondientes.

Los ligamentos involucrados son los que forman la cintura escapular, que comprenden a los ligamentos esternoclaviculares anterior y posterior, interclavicular, costoclavicular, acromioclavicular, coracoclaviculares, conoide, trapezoide, transverso, coracohumeral y glenohumeral superior, medio e inferior. A su vez, los músculos que hacen posibles los movimientos en estudio son: pectoral mayor, dorsal ancho, supraespinoso, subescapular, infraespinoso, redondo menor, redondo mayor, deltoides cabeza anterior, deltoides cabeza media, deltoides cabeza posterior y coracobraquial.
Tabla 4: Nomenclatura de tensores pasivos y activos en la UDB_hombro. Las columnas corresponden al nombre anatómico, la correspondiente nomenclatura asignada en la UDB y los puntos de inserción en los que se conecta cada tensor.

\begin{tabular}{|c|c|c|c|}
\hline $\begin{array}{c}\text { Tipo } \\
\text { tensores }\end{array}$ & Nom. anatómico & $\begin{array}{l}\text { Nom. } \\
\text { UDB }\end{array}$ & $\begin{array}{l}\text { P. de } \\
\text { inser. } \\
\text { conec. }\end{array}$ \\
\hline \multirow{9}{*}{$\begin{array}{l}\text { Tensores } \\
\text { pasivos }\end{array}$} & $\begin{array}{c}\text { Ligamento } \\
\text { acromioclavicular }\end{array}$ & & \multirow{9}{*}{$\begin{array}{l}\text { c2, c3, c4 } \\
\text { h3, h4, h9 }\end{array}$} \\
\hline & L. costoclavicular & & \\
\hline & & L1 & \\
\hline & L. esternoclavicular & L2 & \\
\hline & \multirow{2}{*}{$\begin{array}{l}\text { L. esternoclaviculares } \\
\text { anterior y posterior }\end{array}$} & L4 & \\
\hline & & $\begin{array}{l}\text { L5 } \\
\text { L6 }\end{array}$ & \\
\hline & L. interclavicular & L7 & \\
\hline & & L8 & \\
\hline & $\begin{array}{l}\text { L. conoide y } \\
\text { trapezoide }\end{array}$ & & \\
\hline \multirow{11}{*}{$\begin{array}{l}\text { Tensores } \\
\text { activos }\end{array}$} & M. Pectoral mayor & T12 & e1-h1 \\
\hline & M. Dorsal ancho & T11 & d1- h2 \\
\hline & M. Supraespinoso & T16 & ec1-h3 \\
\hline & M. Subescapular & T19 & ec2-h4 \\
\hline & M. infraespinoso & T17 & ec2-h3 \\
\hline & M. redondo menor & $\mathrm{T} 10$ & ec4-h5 \\
\hline & M. redondo mayor & T13 & ec5-h6 \\
\hline & $\begin{array}{c}\text { M. Deltoides cabeza } \\
\text { anterior }\end{array}$ & T15 & c1-h7 \\
\hline & $\begin{array}{l}\text { M. Deltoides cabeza } \\
\text { media }\end{array}$ & T14 & ec1-h8 \\
\hline & $\begin{array}{l}\text { M. Deltoides cabeza } \\
\text { posterior }\end{array}$ & T18 & ec4-h3 \\
\hline & M. Coracobraquial & T19 & ec5-h10 \\
\hline
\end{tabular}

\section{RESULTADOS}

El proceso desarrollado hasta aquí permitió la generación de una primera clasificación de Unidades Dinámicas de Tensegridad. Esta clasificación responde a criterios morfológicos y se presenta como una tabla que cruza la cantidad de extensiones de cada compresor. La figura 4 da cuenta de una selección de las Unidades Dinámicas Abstractas desarrolladas en este trabajo. Cada una de estas UDAs tiene movimientos potenciales que pueden ser definidos por la combinatoria de los tensores que sean activados, las longitudes de sus extensiones y la posición de los nudos de conexión de los tensores. A su vez, pueden convertirse en células de un tejido complejo o articulaciones de mecanismos específicos.

En la Figura 8 se observan diferentes etapas de la simulación del movimiento de flexión de la UDB_pierna y UDB_hombro. 

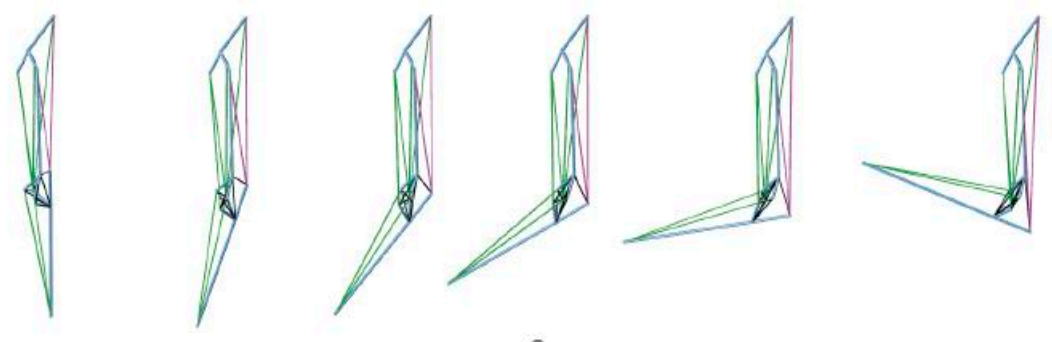

a
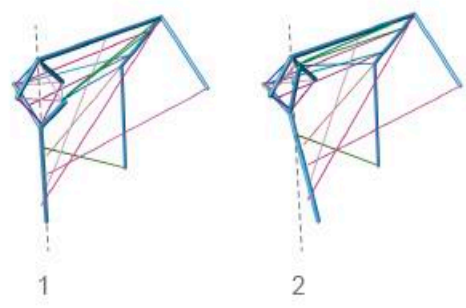

b
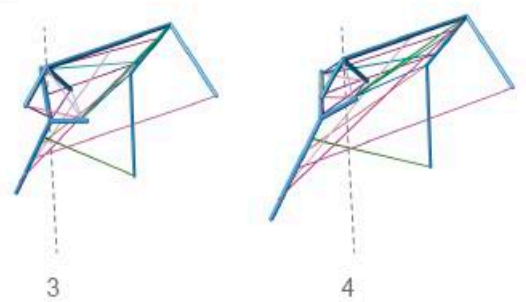

Figura 8: Instancias de simulación de las Unidades Dinámicas Biodiseñadas desarrolladas con base en la pierna y el brazo (derechos). a) UDB_pierna. Secuencia de flexión, b) UDB_hombro. 1. En reposo, 2. Flexión, 3. Extensión, 4. Abducción.

\section{DISCUSIÓN Y CONCLUSIONES}

Las UDAs aquí presentadas pueden convertirse en células de un tejido complejo, articulaciones de mecanismos específicos que pueden ser productos innovadores en sí mismos o presentar alguna ventaja diferencial sobre las articulaciones y mecanismos existentes. Este catálogo pretende ser una primera guía de sugerencias para ser abordada y analizada, en profundidad, según casos específicos de diseño. Esta Clasificación 1.0 de UDAs puede seguir ampliándose al generar células dinámicas con los principios aquí presentados, mayor número de extensiones en los componentes compresores y sus combinaciones, variaciones en las características de los tensores, etc.

Por su parte, las UDBs desarrolladas hasta aquí son un primer acercamiento a la emulación de los movimientos biomecánicos desde un abordaje bioinformado y desarrollada con herramientas digitales propias del diseño. $\mathrm{Si}$ bien, estas propuestas son abstracciones cuya interpretación y detalles son definidos por sus autores, permite observar la complejidad propia de un proceso de bioinformación adaptado desde un abordaje netamente morfológico.

En las UDBs desarrolladas se han estudiado tan sólo algunos movimientos producidos mediante la activación de una sola articulación, en cada caso (rodilla y hombro), incluyendo la mayor cantidad de huesos, músculos y ligamentos asociados en sus movimientos. De esta manera, en los resultados obtenidos en las simulaciones, se observan movimientos similares a los de la biotensegridad modelo. Todo ello logrado en estas interpretaciones tecnomorfológicas luego de haber reducido la complejidad orgánica mediante la fusión de algunos de sus componentes.
En la simulación de la UDB pierna el control fue mayor debido a aspectos morfológicos específicos, como la concentración cuasi-lineal de los componentes, y al hecho que la UDA que compone la articulación (rodilla en este caso), es del tipo 2/3 e incluye una extensión de 3 en la parte correspondiente a la cadera. Estas triangulaciones de tensores junto a la direccionalidad de los mismos, permiten un control más preciso de los movimientos. En la UDB_pierna se aplicó una UDA $2 / 2$ y sus componentes están configurados en un espacio primordialmente bidimensional. Esto suma vectores de fuerza en direcciones transversales a los componentes, haciendo necesario un sistema de control más complejo.

Por su parte, estudiar segmentos aislados también repercute en la performance dinámica del sistema, de tal manera que no es posible obtener la complejidad y fidelidad de los movimientos biológicos en forma completa. Se considera lo realizado un paso preliminar, hacia la compresión y traducción de biotensegridades articulares.

\section{PERSPECTIVAS FUTURAS}

La parametrización de los movimientos debe contemplar la activación simultánea y coordinada de tensores agonistas y antagonistas, de tal manera que respondan de manera más coherente con la biomecánica en cuestión.

La optimización topológica de los componentes es una tarea clave, presente en la agenda de investigación. Se pretende lograr emulaciones más precisas que permitan la fabricación de posibles mecanismos para prótesis, órtesis o incluso, robots con movimientos bioinformados.

La fabricación digital integral de los modelos aquí presentados, junto al estudio experimental de su comportamiento dinámico, será el próximo paso. 
En cuanto al proceso de bioinformación se proyecta incluir a las fascias y las cadenas cinemáticas cerradas, lo cual permitirá, hipotéticamente, lograr movimientos más precisos y una integridad más coherente con estos sistemas complejos.

\section{AGRADECIMIENTOS}

La presente investigación fue realizada dentro del marco del Proyecto de Investigación PIT TRP-03 de la Facultad de Arquitectura, Diseño y Urbanismo de la Universidad de Buenos Aires. Los autores agradecen a las y los pasantes de las carreras de diseño industrial y arquitectura y al Arquitecto Arturo de la Fuente por su apoyo al proyecto como instructor en el área de diseño paramétrico.

Esta investigación ha sido financiada parcialmente por: a) el Proyecto interdisciplinario No. 206201600100004BA con subsidio de la Programación Científica (UBA) 2017 2019 y b) del financiamiento otorgado por la Pontificia Universidad Católica Argentina, Facultad de Ingeniería y Ciencias Agrarias, en el marco del Proyecto Modelos Biomecánicos en conjunto con el desarrollo de dispositivos para la cuantificación del equilibrio humano en individuos normales y/o disfuncionales.

\section{REFERENCIAS}

Aldrich, J. B., Skelton, R. E., \& Kreutz-Delgado, K. (2003, June). Control synthesis for a class of light and agile robotic tensegrity structures. In Proceedings of the 2003 American Control Conference, 2003. (Vol. 6, pp. 5245-5251). IEEE.

Castro-Arenas, Ghersi, Borsoi, \& Miralles (2017). Platonic Tensegrities: dynamic aspects and characterization. In VII Latin American Congress on Biomedical Engineering CLAIB 2016, Bucaramanga, Santander, Colombia, October 26th28th, 2016 (pp. 264-267). Springer, Singapore.

Cretu, S. M. (2009). Tensegrity Concept-From Natural Systems to Robots. In Proceedings of EUCOMES 08 (pp. 549-557). Springer, Dordrecht.

Cretu, S. M. (2011). Innovative design in tensegrity field. Procedia Engineering, 9, 261-269.

Emmerich, D. G. (1992). Stable Simplex. An introduction to the resistance of formes. In Proceedings of the First International Seminar on Structural Morphology, Montpellier.

Friesen, J., Pogue, A., Bewley, T., de Oliveira, M., Skelton, R., \& Sunspiral, V. (2014, May). DuCTT: A tensegrity robot for exploring duct systems. In 2014 IEEE International Conference on Robotics and Automation (ICRA) (pp. 42224228). IEEE.

Fuller, B. R., \& Applewhite, E. J. (1975). Synergetics: explorations in the geometry of thinking. Charles Scribner's Sons, New York, 876.

Fuller, B. (1961). Tensegrity. Portfolio Artnews Annual, 4, 112-127.

González, A., \& Luo, A. (2019, June). Design and Control of a Tensegrity-Based Robotic Joint. In IFToMM World Congress on Mechanism and Machine Science (pp. 2631-2640). Springer, Cham.

Hensel, M., \& Menges, A. (2008). Designing Morpho-Ecologies: Versatility and Vicissitude of Heterogeneous Space. Architectural Design, 78(2), 102-111.

Ingber, D. E. (1993). Cellular tensegrity: defining new rules of biological design that govern the cytoskeleton. Journal of cell science, 104(3), 613-627.

Ingber, D. E. (1998). The architecture of life. Scientific American, 278(1), $48-5 \mathrm{n} 7$
Ingber, D. E. (2003). Tensegrity I. Cell structure and hierarchical systems biology. Journal of cell science, 116(7), 1157-1173. Accesible en https://jcs.biologists.org/content/116/7/1157.

Ingber, D. E. (2018). Tensegrity as the architecture of life. In Proceedings of IASS Annual Symposia (Vol. 2018, No. 27, pp. 1-4). International Association for Shell and Spatial Structures (IASS).

louguina, A., Dawson, J. W., Hallgrimsson, B., \& Smart, G. (2014). Biologically informed disciplines: A comparative analysis of bionics, biomimetics, biomimicry, and bio-inspiration among others. International Journal of Design \& Nature and Ecodynamics, 9(3), 197-205.

Jung, E., Ly, V., Cessna, N., Ngo, M. L., Castro, D., SunSpiral, V., \& Teodorescu, M. (2018). Bio-inspired tensegrity flexural joints. In 2018 IEEE International Conference on Robotics and Automation (ICRA) (pp. 1-6). IEEE

Khazanov, M., Humphreys, B., Keat, W., \& Rieffel, J. (2013, September). Exploiting dynamical complexity in a physical tensegrity robot to achieve locomotion. In Artificial Life Conference Proceedings 13 (pp. 965-972). One Rogers Street, Cambridge, MA 02142-1209 USA journals-info@ mit. edu: MIT Press.

Lessard, S., Bruce, J., Jung, E., Teodorescu, M., SunSpiral, V., \& Agogino, A. (2016, May). A lightweight, multi-axis compliant tensegrity joint. In 2016 IEEE International Conference on Robotics and Automation (ICRA) (pp. 630-635). IEEE.

Levin, S.M., (1982) Continuous Tension, Discontinuous Compression. A Model for Biomechanical Support of the Body. Bulletin of Structural Integration, Rolf Institute, Bolder. pp.3133.

Levin S.M., (1991) Primordial Structure. Proc. vol 2, 34th An.Meet. Int. Soc. Syst. Sci., Portland.

Levin, S.M., (2006) Tensegrity, The New Biomechanics Textbook of Musculoskeletal Medicine ed. Hutson M \& Ellis R (Oxford: Oxford University Press) pp $69-80$.

Motro, R. (2003). Tensegrity: structural systems for the future. Elsevier.

Pajunen, K. M. (2020). Dynamics of Lightweight TensegrityInspired Metamaterials Fabricated with 3D-Printing (Doctoral dissertation, California Institute of Technology).

Reissig, P. (2012). Tecno-morfología como estrategia de diseño. Tesis Doctoral defendida en 2012, Publicado por el Instituto de la Espacialidad Humana, FADU, Universidad de Buenos Aires, ISBN: 978950-29-1795-5.

Rimoli, J. J., \& Pal, R. K. (2017). Mechanical response of 3dimensional tensegrity lattices. Composites Part B: Engineering, 115, 30-42.

Scarr, G. (2014). Biotensegrity. Handspring Publishing, United Kingdom.

Skelton, R. E., Helton, W. J., \& Adhikari, R. (1998). Mechanics of Tensegrity Beams, UCSD, Structural Systems \& Contr. Lab., Rep, (1998).

Skelton, R. (2005). Dynamics and control of tensegrity systems. In IUTAM symposium on vibration control of nonlinear mechanisms and structures (pp. 309-318). Springer, Dordrecht.

Swanson, R. L. (2013). Biotensegrity: a unifying theory of biological architecture with applications to osteopathic practice, education, and research-a review and analysis. The Journal of the American Osteopathic Association, 113(1), 3452.

SunSpiral V., Agogino A., \& Atkinson D. (2015) Super Ball Bot Structures for Planetary Landing and Exploration, NIAC Phase 2 - Final Report. NASA Innovative Advanced Concepts (NIAC) Program. NASA Ames Research Center. Intelligent Systems Division. Recuperado de NASA Technical Report Server (NTRS) https://go.nasa.gov/3ePQOUD. 